\title{
Evaluation of an arboviral syndrome query used in Maricopa County, Arizona
}

\author{
Kaitlyn Sykes ${ }^{\star 1,2}$, Rasneet S. Kumar ${ }^{1}$, Melissa Kretschmer ${ }^{1}$ and Jessica R. White ${ }^{1}$ \\ ${ }^{1}$ Maricopa County Department of Public Health, Phoenix, AZ, USA; ${ }^{2}$ CSTE Applied Epidemiology Fellowship, Atlanta, GA, USA
}

\section{Objective}

To evaluate Arizona's arboviral syndromic surveillance protocol in Maricopa County.

\section{Introduction}

Timely identification of arboviral disease is key to prevent transmission in the community, but traditional surveillance may take up to 14 days between specimen collection and health department notification. Arizona state and county health agencies began monitoring National Syndromic Surveillance Program BioSense 2.0 data for patients infected with West Nile virus (WNV), St. Louis encephalitis virus (SLEV), chikungunya, or dengue virus in August 2015. Zika virus was added in April 2016. Our novel methods were presented at the International Society for Disease Surveillance 2015 Annual Conference. [1] Twice per week, we queried patient records from 15 Maricopa County BioSense-enrolled emergency department and inpatient hospitals for chief complaint keywords and discharge diagnosis codes. Our "Case Investigation Decision Tree" helped us determine whether records had a high or low degree of evidence for arboviral disease and necessitated further investigation. This study evaluated how Arizona's protocol for conducting syndromic surveillance compared to traditional arboviral surveillance in terms of accuracy and timeliness in Maricopa County from August 2015 through December 2016.

\section{Methods}

We followed guidelines from the Centers for Disease Control and Prevention (CDC) to evaluate two major attributes of the protocol: accuracy [measured as positive predictive value (PPV) and sensitivity] and timeliness. [2] Arizona's Medical Electronic Disease Surveillance Intelligence System (MEDSIS) was considered the "gold standard" system and BioSense was the test system. PPV was calculated as the proportion of records identified by BioSense that were reported to MEDSIS, regardless of final case classification. Sensitivity was the proportion of confirmed or probable cases in MEDSIS identified by BioSense. Though not all MEDSIS cases were seen at BioSensereporting facilities, the sensitivity demonstrates how each query contributed to arboviral surveillance overall. We assessed timeliness in two ways: (1) the difference between the date when keywords or diagnosis codes were first identified by BioSense and the date the same patient was first reported to MEDSIS; and (2) the difference between the date the BioSense record was first reviewed by the Maricopa County Department of Public Health (MCDPH) syndromic surveillance team and the date the same patient was first investigated through MEDSIS by the MCDPH disease investigators. We assessed whether timeliness was affected by the method in which a record was identified in BioSense (i.e., chief complaint keyword or discharge diagnosis code).

\section{Results}

The arboviral syndromic surveillance queries identified 62 records during the evaluation period (Table). For each arboviral query, the proportion of BioSense records that were also reported through MEDSIS ranged from $25.0 \%$ to $32.4 \%$, except chikungunya, which had a PPV of $0 \%$. BioSense records that had a high degree of evidence for arboviral disease tended to have a higher PPV compared to those with low evidence. BioSense records that were not already reported to MEDSIS met neither clinical nor exposure criteria for the arboviral diseases and were not deemed a public health risk. The sensitivities of the WNV and Zika queries to detect confirmed or probable cases in MEDSIS were $8.2 \%$ and 5.6\%, respectively, while SLEV, chikungunya, and dengue queries were $0 \%$. On average, patients were reported to MEDSIS 7 days prior to BioSense identifying keywords or diagnosis codes. In addition, MEDSIS cases were investigated by MCDPH disease investigators 10 days prior to MCDPH syndromic surveillance team review of BioSense records, on average. The average time between MEDSIS report date and BioSense identification date was shorter for BioSense records identified by chief complaint keywords than by diagnosis codes ( 4 and 8 days after MEDSIS, respectively).

\section{Conclusions}

Arizona's arboviral syndromic surveillance protocol provided MCDPH with situational awareness, but BioSense data were not available more quickly than traditional mandated reporting. Through this process, we reviewed patient records that mentioned arboviral diseases and confirmed that these reportable conditions were captured in our traditional surveillance system. The decision tree was effective at prioritizing records for further investigation. Timeliness may be improved by updating the queries to include more chief complaint keywords and reviewing BioSense more than twice per week. MCDPH plans to evaluate Arizona's updated arboviral syndromic surveillance protocol, which was adapted for BioSense Platform's Electronic Surveillance System for Early Notification of Communitybased Epidemics (ESSENCE).

\begin{tabular}{|c|c|c|}
\hline & $\begin{array}{l}\text { Positive predictive value of query = } \\
\text { Records reported to MEDSIS (any class) }\end{array}$ & $\begin{array}{c}\text { Sensitivity of query = } \\
\text { Records identified by BioSense }\end{array}$ \\
\hline & Total records identified by BioSense & Total confirmed or probable cases in MEDSIS \\
\hline WNV & $12 / 37(32.4 \%)$ & $7 / 85 \quad(8.2 \%)$ \\
\hline High evidence & $11 / 33(33.3 \%)$ & -. \\
\hline Low evidence & $1 / 4(25.0 \%)$ & -- \\
\hline SLEV & $1 / 4(25.0 \%)$ & $0 / 4 \quad(0.0 \%)$ \\
\hline High evidence & $1 / 4(25.0 \%)$ & -- \\
\hline Low evidence & $-10(\mathrm{~N} / \mathrm{A})$ & -- \\
\hline Chikungunya & $0 / 1(0.0 \%)$ & $0 / 13 \quad(0.0 \%)$ \\
\hline High evidence & $0 / 1(0.0 \%)$ & -- \\
\hline Low evidence & $-10(\mathrm{~N} / \mathrm{A})$ & -- \\
\hline Dengue & $3 / 11(27.3 \%)$ & $0 / 18 \quad(0.0 \%)$ \\
\hline High evidence & $3 / 9(33.3 \%)$ & - \\
\hline Low evidence & $0 / 2(0.0 \%)$ & -- \\
\hline Zika & $3 / 10(30.0 \%)$ & $2 / 36 \quad(5.6 \%)$ \\
\hline High evidence & $1 / 5(20.0 \%)$ & -- \\
\hline Low evidence & $2 / 5(40.0 \%)$ & -- \\
\hline
\end{tabular}
virus: WNY, West Nile virus

\section{Keywords}

Arboviral; Evaluation; Surveillance

\section{Acknowledgments}

This study was supported in part by an appointment to the Applied Epidemiology Fellowship Program administered by the Council of State and Territorial Epidemiologists and funded by CDC Cooperative Agreement Number 1U38OT000143-04. 
References

1. White, J. R., Imholte, S., \& Collier, K. (2016). Using Syndromic Surveillance to Enhance Arboviral Surveillance in Arizona. Online $J$ Public Health Inform, 8(1), e81.

2. German, R. R., et al. (2001). Updated guidelines for evaluating public health surveillance systems: recommendations from the Guidelines Working Group. MMWR Recomm Rep, 50(RR-13), 1-35.

\section{*Kaitlyn Sykes}

E-mail: kaitlynsykes@mail.maricopa.gov 